\title{
Cucurbitacin D impedes gastric cancer cell survival via activation of the iNOS/NO and inhibition of the Akt signalling pathway
}

\author{
YAN ZHEN ZHANG, CHUN FENG WANG and LIAN FENG ZHANG
}

Department of Gastroenterology, The First Affiliated Hospital of Zhengzhou University, Zhengzhou, Henan 450052, P.R. China

Received October 14, 2017; Accepted March 21, 2018

DOI: $10.3892 / o r .2018 .6361$

\begin{abstract}
Cucurbitacin D (CuD), isolated from plants from the Cucurbitaceae family, is a potential antitumour agent since it inhibits proliferation, migration and metastasis of cancer cells. Despite CuD antitumour activity in cancer cells, the effects of $\mathrm{CuD}$ on gastric cancer cell lines remain unclear. The present study aimed to investigate the effects of $\mathrm{CuD}$ on gastric cancer cell growth and death. Human gastric cancer cell lines (AGS, SNU1 and Hs746T) were cultured and treated with different concentrations of $\mathrm{CuD}(0,0.25,0.5,1$ and $2 \mu \mathrm{M})$. Cell proliferation was assessed using Cell Counting Kit-8 assay. Oxidative stress was evaluated by generation of reactive oxygen species (ROS). Cell apoptosis was assessed by terminal deoxynucleotidyl transferase 2'-deoxyuridine-5'-triphosphate nick-end labelling (TUNEL) staining. Levels of intracellular $\mathrm{Ca}^{2+}$ and adenosine triphosphate (ATP) were also assessed. In the present study, CuD effectively inhibited cell proliferation, triggered ROS generation and induced apoptosis in gastric cancer cells (AGS, SNU1 and Hs746T). Treatment with $\mathrm{CuD}$ increased intracellular $\mathrm{Ca}^{2+}$ and ATP levels. $\mathrm{CuD}$ also stimulated the expression of inducible nitric oxide synthase (iNOS), which augmented nitric oxide production. In addition, $\mathrm{CuD}$ activated the mitochondrial apoptosis pathway, which increased the expression of Bax and the release of cleaved caspace-9 (C-caspase-9) and cytochrome $c$, decreased the expression of B-cell lymphoma 2 (Bcl-2). The mechanism of action of $\mathrm{CuD}$ involved the regulation of the protein kinase $\mathrm{B} / \mathrm{mechanistic}$ target of rapamycin (Akt/mTOR) pathway. We confirmed the effects of $\mathrm{CuD}$ on gastric tumours via an in vivo xenograft gastric tumour model. In conclusion, $\mathrm{CuD}$ inhibited Akt and activated the iNOS pathway, leading to higher ROS and nitric oxide production, which accelerated gastric cancer cell apoptosis.
\end{abstract}

Correspondence to: Dr Yan Zhen Zhang or Professor Lian Feng Zhang, Department of Gastroenterology, The First Affiliated Hospital of Zhengzhou University, 1 Jianshedong Road, Zhengzhou, Henan 450052, P.R. China

E-mail: yanzhenzhang2005@126.com

E-mail: zlfzzuxh@163.com

Key words: cucurbitacin D, gastric cancer, Akt, iNOS, apoptosis

\section{Introduction}

Although the incidence of gastrointestinal cancer is lower than that of other cancers, it is the second leading cause of cancer-related deaths worldwide (1). Gastric cancer begins in the inner lining of cells, causing abnormal hyperplasia that leads to ulceration, inflammation and ultimately tumour formation (2). More than 50\% of distal gastric cancer patients can be cured, however, early diagnosis accounts for only $10-20 \%$ of all cases (3). The mortality rate of gastric cancer has declined significantly with the latest advances in diagnosis and treatment. However, most patients are diagnosed at an advanced stage, which is difficult to cure (4). For patients with node-positive (T1 N1) and muscle-invasive (T2 N0) disease, radiotherapy and chemotherapy maybe the most effective treatment measures. However, patients receiving radiotherapy and chemotherapy may have problems with chemoresistance and toxic side-effects. Thus, further studies are needed in order to develop new treatment strategies.

Increasingly, researchers are focusing on naturally occurring compounds from dietary sources (5). Cucurbitacins, which are found in plants from the Cucurbitaceae family, are tetracyclic triterpenes. Cucurbitacins exhibit moderate to high toxicity, but they contain structural properties that may aid in future chemotherapy modalities. These properties make them potential antitumour agents $(5,6)$. Cucurbitacin D (CuD; Fig. 1) is a member of the Cucurbitaceae family. Studies have reported the anticancer activity of $\mathrm{CuD}$ in various cancer models $(5,7,8)$. $\mathrm{CuD}$ has been reported to inhibit the growth of cervical cancer cells (5) and doxorubicin-resistant human breast carcinoma cells (8). In addition, $\mathrm{CuD}$ has been found to suppress the heat shock protein 90 (HSP90) chaperone machinery (9). These data indicated that $\mathrm{CuD}$ potentially affects gastric cancer. The aim of the present study was to investigate the in vitro and in vivo effects of $\mathrm{CuD}$ on gastric cancer growth and apoptosis.

The progression of gastric cancer occurs through various oncogenic pathways such as the nuclear factor- $\kappa \mathrm{B}$, phospho inositide 3-kinase/protein kinase B (PI3K/Akt) and Wnt/ $\beta$-catenin pathways (10). Among these, the PI3K/Akt pathway is an important signalling pathway that regulates cell survival, growth, metabolism and chemotherapy resistance (11). Gastric cancer is characterized by a high rate of somatic cell turnover via the PI3K/Akt pathway, which indicates that PI3K/Akt may be an effective therapeutic target (12). In the present study, we observed that $\mathrm{CuD}$ effectively triggered 
gastric cancer cell apoptosis by inhibiting Akt and activating the inducible nitric oxide synthase (iNOS) pathway.

\section{Materials and methods}

Cells and drugs. AGS, SNU1 and Hs746T cell lines were purchased from Corbioer Company (Nanjing, China). Shanghai Winherb Medical S\&T Development Co. Ltd. (Shanghai, China) provided purified $\mathrm{CuD}$. All the primary antibodies including those for iNOS (diluted at 1:1,000; cat. no. 13120), Bax (diluted at 1:1,000; cat. no. 2722), B-cell lymphoma 2 (Bcl-2; cat. no. 2870), C-caspase-9 (diluted at 1:1,000; cat. no. 9509P), cytochrome $c$ (diluted at 1:1,000; cat. no. 4272), phosphorylated and total Akt (diluted at 1:1,000; cat. no. 4060/4691), mechanistic target of rapamycin (mTOR, cat. no. 2971/2983), sr6 (diluted at 1:1,000; cat. no. 9204/2708) and glyceraldehyde 3-phosphate dehydrogenase (GAPDH; diluted at 1:1,000; cat. no. 5174) were obtained from Cell Signaling Technology, Inc. (Danvers, MA, USA). The Akt activator SC79 was obtained from Sigma-Aldrich (Merck KGaA, Darmstadt, Germany.) The iNOS inhibitor L-canavanine was purchased from MedChemExpress (Monmouth Junction, NJ, USA).

Cell proliferation assay. Cell proliferation and viability were assessed by Cell Counting Kit-8 (CCK-8) assay (Beyotime Institute of Biotechnology, Haimen, China). Cells were seeded into a 96-well plate. After the cells were treated with $\mathrm{CuD}$, CCK-8 solution $(10 \mu \mathrm{l})$ was added to each well. After $4 \mathrm{~h}$ of incubation, an enzyme-linked immunosorbent assay (ELISA) (Synergy HT; BioTek Instruments, Inc., Winooski, VT, USA) was used to the determine absorbance at $450 \mathrm{~nm}$.

TUNEL staining. Apoptosis was detected by a terminal deoxynucleotidyl transferase 2'-deoxyuridine-5'-triphosphate nick-end labelling (TUNEL) assay (ApopTag Plus Fluorescein In Situ Apoptosis Detection kit; Millipore, Darmstadt, Germany). After washing with phosphate-buffered saline (PBS; Gibco, Grand Island, NY, USA), the cells were fixed with $1 \%$ paraformaldehyde in PBS. TUNEL reagents (EMD Millipore, Billerica, MA, USA) were used to stain the apoptotic cells and 4',6-diamidino-2-phenylindole (DAPI; Invitrogen; Life Technologies Carlsbad, CA, USA) was used to stain the DNA. A microscope (Olympus BX51TRF; Olympus Corp., Tokyo, Japan) was used to analyse and count positive cells.

ROS generation. Reactive oxygen species (ROS) were detected with a ROS Assay kit (Cell Biolabs, Inc., San Diego, CA, USA). A cell-permeable fluorogenic probe 2',7'-dichlorodihydrofluorescin diacetate (DCFH-DA) was used to label ROS. A fluorometric plate reader (Thermo Fisher Scientific, Inc., Waltham, MA, USA) was used to quantify fluorescence at $480 / 530 \mathrm{~nm}$.

ATP level. The levels of ATP were detected by an ATP assay kit (S0026; Beyotime Institute of Biotechnology). After the cells were treated with $\mathrm{CuD}$, cells lysates were collected and incubated with ATP detection solution $(100 \mu 1)$. A fluorometric plate reader was used to determine the levels of ATP. Standard curve method was used to calculate the ATP concentration in each group.

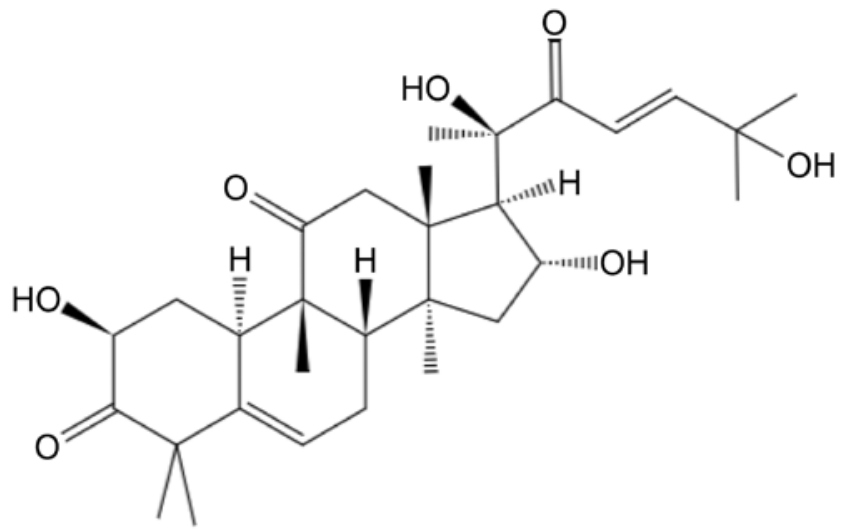

Figure 1. Chemical structure of cucurbitacin $\mathrm{D}(\mathrm{CuD})$ in gastric cancer cell lines.

Intracellular $\mathrm{Ca}^{2+}$ level. The level of intracellular $\mathrm{Ca}^{2+}$ was assessed using Fluo-3/AM (S1056; Beyotime Institute of Biotechnology). After treatment, D-Hanks balanced salt solution (D-HBBS; Jinuo Co., Ltd., Shanghai, China) (without $\mathrm{Ca}^{2+}$, $\mathrm{Mg}^{2+}$ and phenol red) was used to wash cells. Then, cells were incubated with Fluo-3/AM $(5 \mu \mathrm{M})$ for $60 \mathrm{~min}$ at $37^{\circ} \mathrm{C}$. After being washed in D-HBBS for $30 \mathrm{~min}$, a microplate reader was used to determine the fluorescent absorbance value, with representative intracellular $\mathrm{Ca}^{2+}$ levels.

Nitric oxide $(\mathrm{NO})$ production. NO production was detected using Griess method kit (S0021; Beyotime Institute of Biotechnology) according to the instructions of the NO assay kit.

Western blot analysis. Cell lysates were collected using RIPA lysis buffer. Total protein $(50 \mu \mathrm{g})$ was used for SDS-PAGE. After being transferred into immobilon-FL transfer membranes (IPFL00010; Millipore, Billerica, MA, USA), proteins were incubated with the primary antibody overnight at $4^{\circ} \mathrm{C}$, followed by incubation with secondary antibodies for $1 \mathrm{~h}$. A two-coloured infrared imaging system (Odyssey; LI-COR Biosciences, Lincoln, NE, USA) was used to scan. GAPDH protein was used as the reference protein.

Xenograft tumour model. All animal experiments were performed according to the National Institutes of Health Guide for the Care and Use of Laboratory Animals (NIH Publications no. 8023, revised 1978). All animal experiments were approved by the Committee on the Use of Live Animals of The First Affiliated Hospital of Zhengzhou University. Male BALB/c-nu/nu nude mice (6-8 weeks) were purchased from the Beijing HFK Bioscience and housed in specific pathogen-free conditions under approved institutional animal care and use protocols. Mice received injection of $1 \times 10^{6}$ AGS cell suspension. After reaching $\sim 200 \mathrm{~mm}^{3}$ tumour volume, the mice were treated with $\mathrm{CuD}$ (intraperitoneal injection, $1 \mathrm{mg} / \mathrm{kg}$, once daily), SC79 (intraperitoneal injection, $20 \mathrm{mg} / \mathrm{kg}$, once daily) or combination of $\mathrm{CuD}$ with $\mathrm{SC} 79$ for 20 days. The tumor size was measured by vernier caliper measurement of the tumor size and the short diameter. Tumour volumes were calculated using the following formula: Length $\mathrm{x}$ width $^{2} \mathrm{x} 0.5236$. 
A
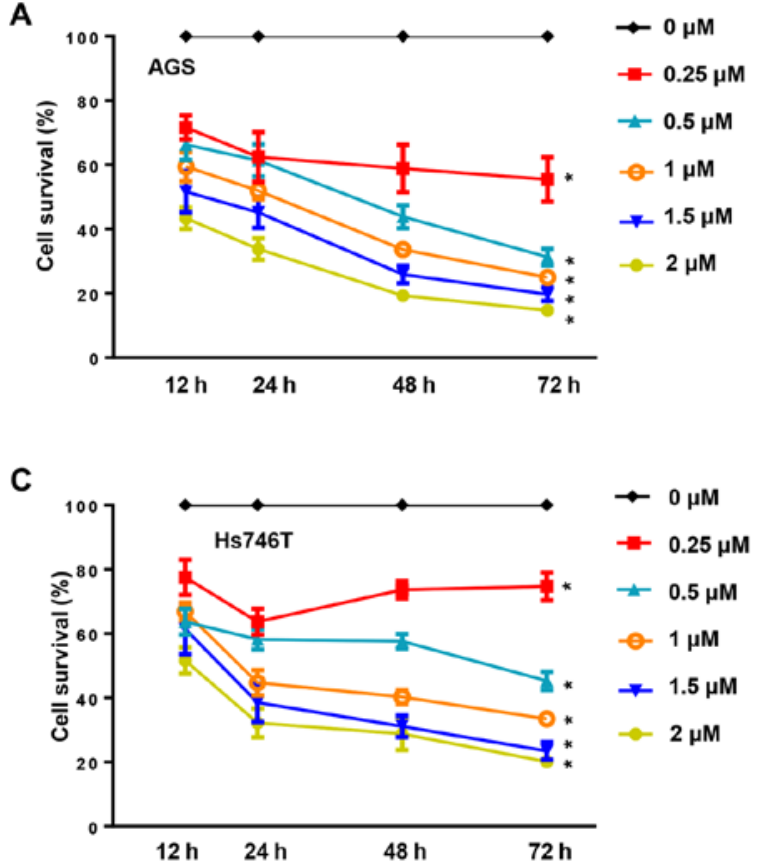

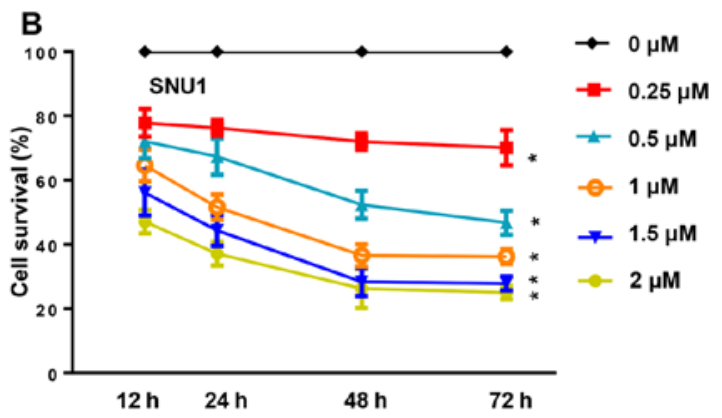

Figure 2. Cucurbitacin $\mathrm{D}(\mathrm{CuD})$ effectively inhibits proliferation in gastric cancer cell lines. The effect of CuD $(0.25,0.5,1,1.5$ and $2 \mu \mathrm{M})$ on cell proliferation in different gastric cancer cell lines: (A) AGS, (B) SNU1 and (C) Hs746T cell line. ${ }^{*} \mathrm{P}<0.05$, compared to the control (0 $\left.\mu \mathrm{M}\right)$ group.
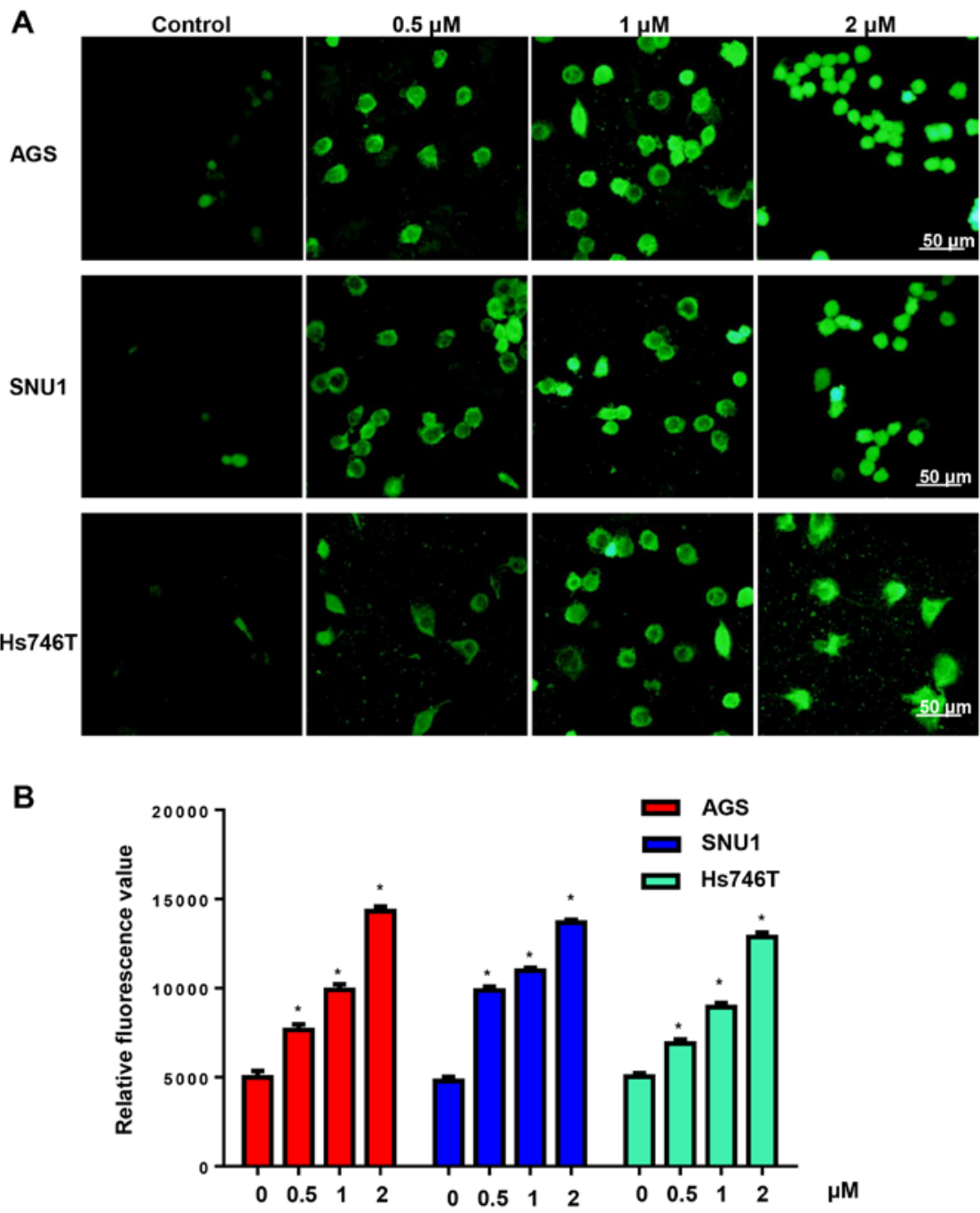

Figure 3. Cucurbitacin $\mathrm{D}(\mathrm{CuD})$ effectively increases ROS generation. $\mathrm{CuD}(0.5,1$ and $2 \mu \mathrm{M})$ induction of reactive oxygen species (ROS) generation in human gastric cancer cell lines as assessed by DCFH-DA. (A) Representative image, (B) representative quantitative result; * $\mathrm{P}<0.05$ compared to the control group. 

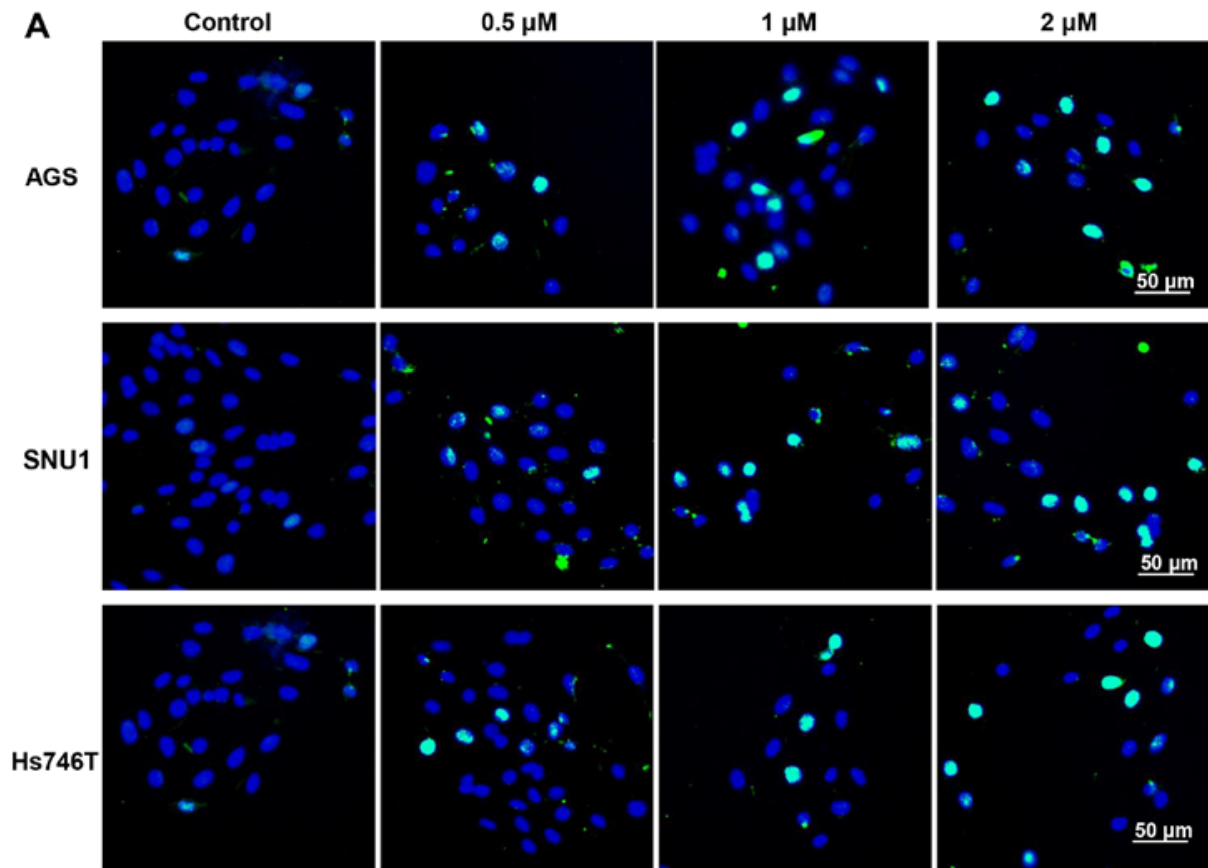

B
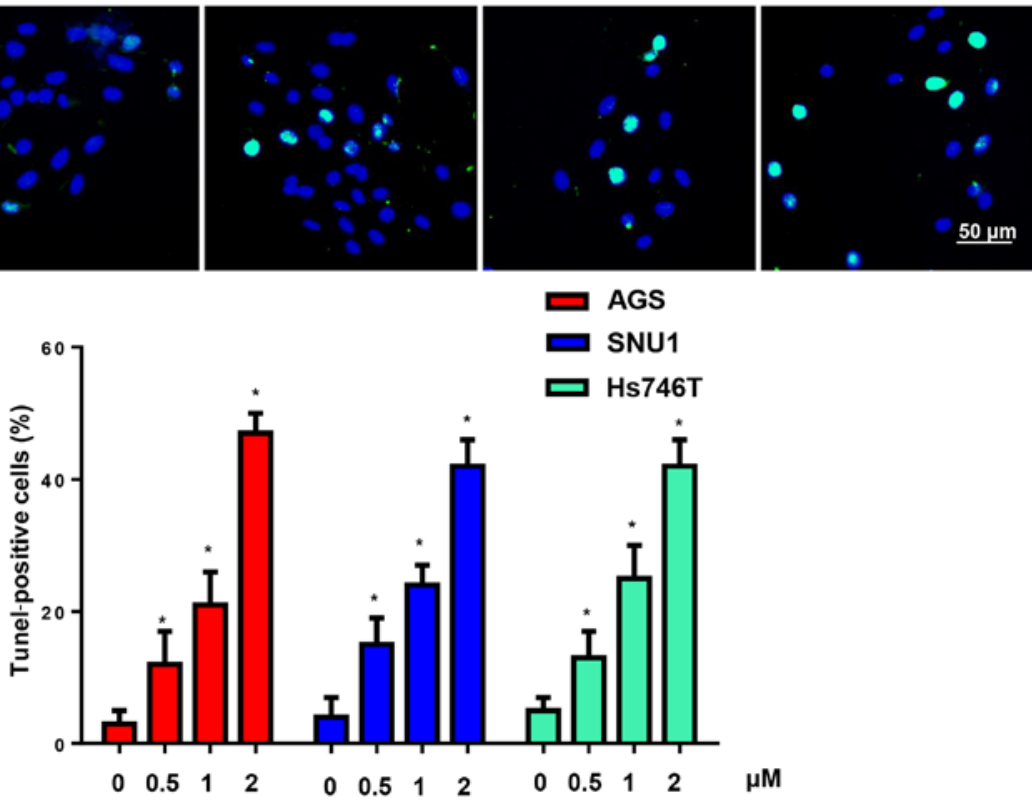

Figure 4. Effect of cucurbitacin $\mathrm{D}(\mathrm{CuD})$ on gastric cancer cell apoptosis. TUNEL staining for the assessment of apoptosis in human gastric cancer cell lines after treatment with $\mathrm{CuD}(0.5,1$ and $2 \mu \mathrm{M})$. (A) Representative image, (B) representative quantitative result; " $\mathrm{P}<0.05$ compared with the control group.

Statistical analysis. We used the SPSS 17.0 (SPSS, Inc.,Chicago, IL, USA) for statistical analysis. All data were expressed as the mean \pm standard deviation. The difference among groups was assessed by analysis of variance (ANOVA). The difference between groups was assessed by Student's t-test. We considered $\mathrm{P}<0.05$ to indicate a statistically significant difference.

\section{Results}

Effects of $C u D$ on gastric cancer cell proliferation. Under normal conditions, human gastric cancer cell lines (AGS, SNU1 and Hs746T) have a fast growth rate. In the present study, cancer cell proliferation was inhibited in a dose- and time-dependent manner when the cells were incubated with $\mathrm{CuD}(0.25,0.5,1,1.5$ and $2 \mu \mathrm{M})$ for $12,24,48$ and $72 \mathrm{~h}$ (Fig. 2). The anti-growth effect of $\mathrm{CuD}$ was more significant in the AGS cell line, indicating that the AGS cell line was more sensitive to $\mathrm{CuD}$.

CuD increases ROS generation in gastric cancer cells. Increasing the generation of ROS is an effective antitumour property of the cucurbitacin family. We detected ROS generation after cells were treated with $\mathrm{CuD}(0.5,1$ and $2 \mu \mathrm{M})$ for $24 \mathrm{~h}$. We found that $\mathrm{CuD}$ increased the ROS levels of gastric cancer cell lines in a dose-dependent manner (Fig. 3).

Effects of CuD on gastric cancer cell apoptosis. Subsequently, TUNEL staining was used to determine the effect of $\mathrm{CuD}$ on gastric cancer cell apoptosis. After incubation with $\mathrm{CuD}(0.5$, 1 and $2 \mu \mathrm{M}$ ) for $24 \mathrm{~h}$, the gastric cancer cells had a high ratio of apoptosis-positive to apoptosis-negative cells, which was dose-dependent (Fig. 4).

$\mathrm{CuD}$ increases intracellular $\mathrm{Ca}^{2+}, \mathrm{ATP}$ and $\mathrm{NO}$ concentration. Increased $\mathrm{Ca}^{2+}$ fluxion and generation of ATP are closely related to cell apoptosis. In the present study, intracellular $\mathrm{Ca}^{2+}$ and ATP generation were assessed by their fluorescence absorbance values. Our results revealed that incubation of AGS cells with $\mathrm{CuD}(0.5,1,1.5$ and $2 \mu \mathrm{M})$ for $12,24,48$ and $72 \mathrm{~h}$ increased the levels of intracellular $\mathrm{Ca}^{2+}$ (Fig. 5A) and ATP (Fig. 5B) in a dose- and time-dependent manner. Intracellular nitric oxide $(\mathrm{NO})$ production in the culture media 
A

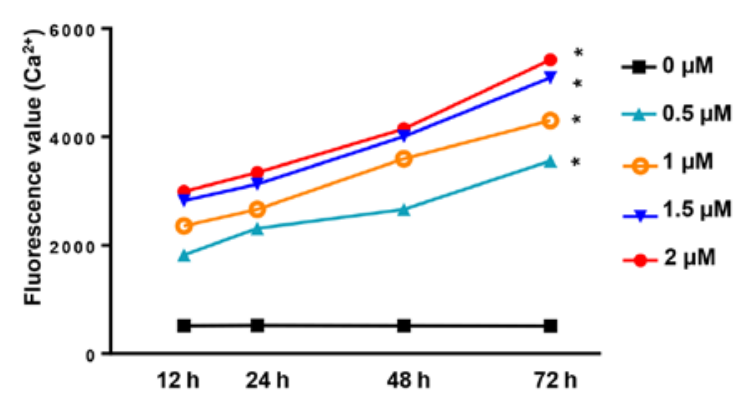

B

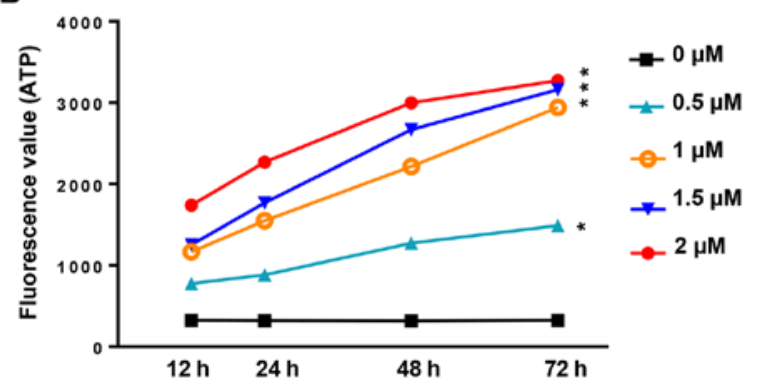

C

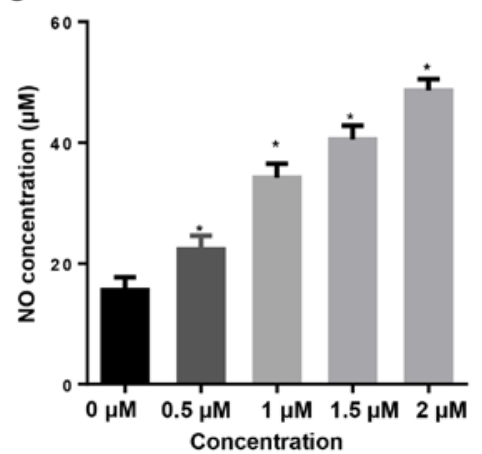

D

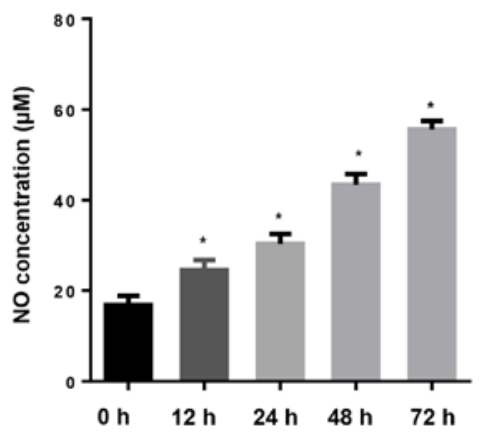

Figure 5. Cucurbitacin D (CuD) increases intracellular $\mathrm{Ca}^{2+}$, ATP and nitric oxide (NO) production. (A and B) Levels of intracellular Ca ${ }^{2+}$ and ATP in CuD-treated AGS cells. Statistical variation of dose-dependent $(0.5,1,1.5$ and $2 \mu \mathrm{M})$ and time-dependent $(12,24,48$ and $72 \mathrm{~h})$ generation of (A) intracellular $\mathrm{Ca}^{2+}$ and (B) ATP. (C and D) Level of intracellular NO in CuD-treated AGS cells. The statistical results of (C) dose-dependent $(0.5,1,1.5$ and $2 \mu \mathrm{M})$ and (D) time-dependent $(12,24,48$ and $72 \mathrm{~h})$ inhibition of $\mathrm{NO}$; ${ }^{*} \mathrm{P}<0.05$ compared to the control group.

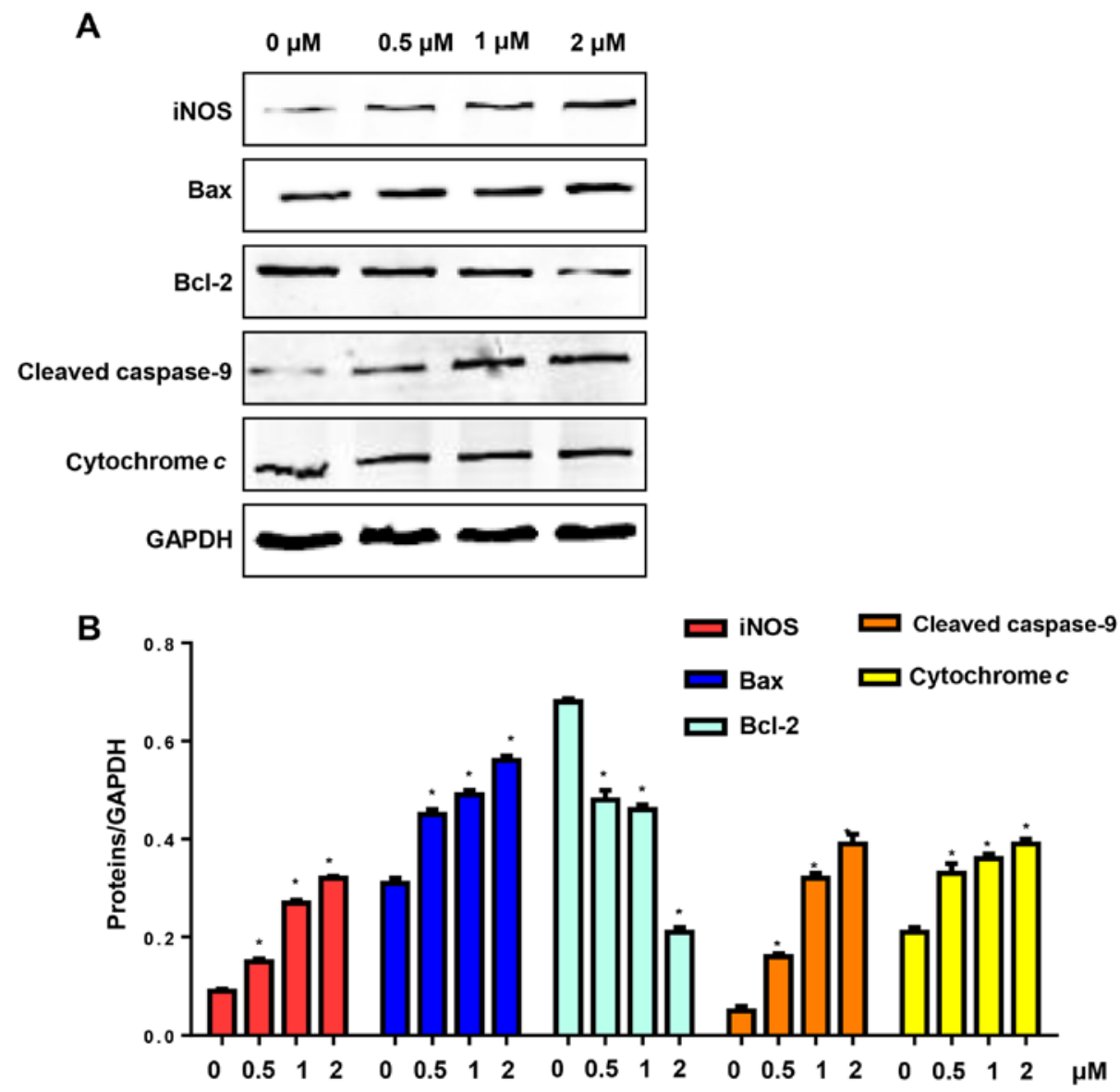

Figure 6. Cucurbitacin $\mathrm{D}(\mathrm{CuD})$ activates the mitochondrial pathway. (A) Western blot analysis of the expression of iNOS and mitochondrial pathway-associated proteins including Bax, Bcl-2, C-Caspase-9 and cytochrome $c$ in AGS gastric cancer cells after treatment with CuD $(0.5,1$ and $2 \mu \mathrm{M})$ for $24 \mathrm{~h}$. (B) The expression levels of the above-mentioned proteins in a bar graph. ${ }^{*} \mathrm{P}<0.05$ compared with the control group. 
A

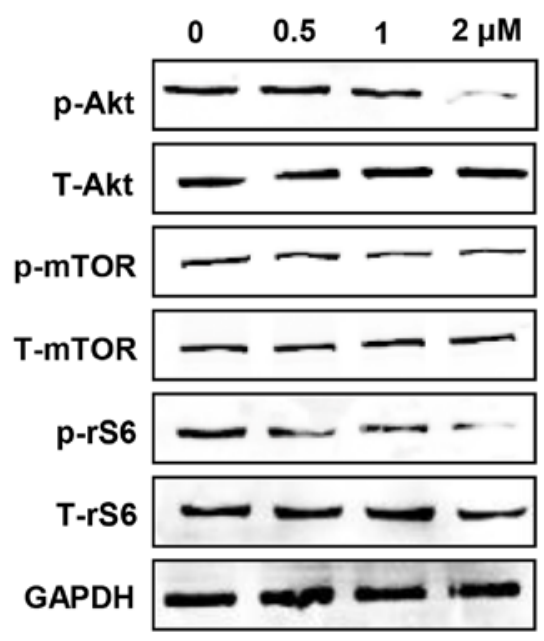

B

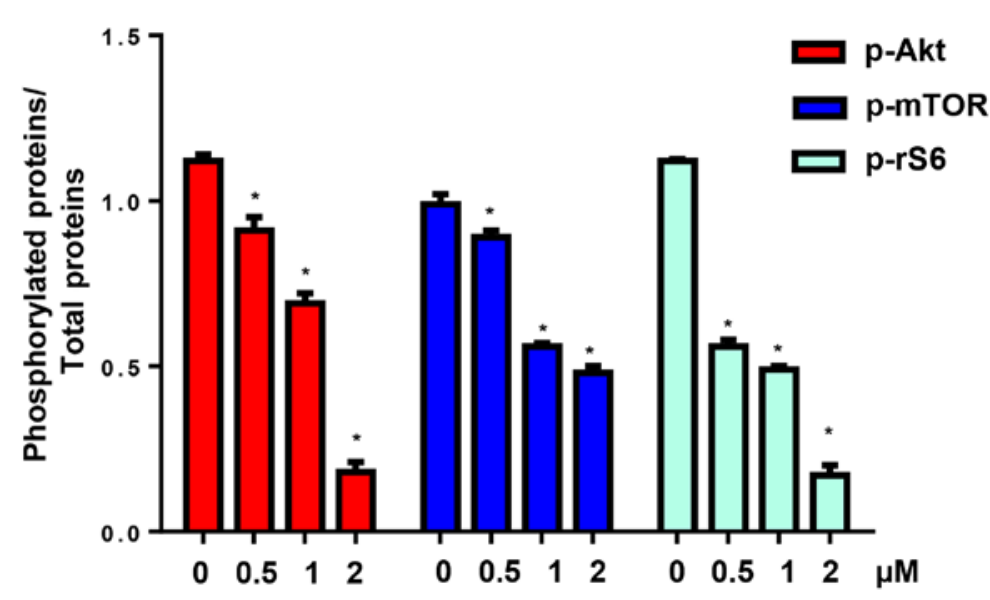

Figure 7. Cucurbitacin D (CuD) inhibits Akt mediated pathway in gastric cancer. (A and B) The expression of phosphorylated and total Akt, mTOR, rs6 in AGS gastric cancer cells after treatment with $\mathrm{CuD}(0.5,1$ and $2 \mu \mathrm{M})$ for $24 \mathrm{~h} .{ }^{*} \mathrm{P}<0.05$ compared to the control group.

was assessed as nitrite concentration. We observed that incubation of AGS cells with $\mathrm{CuD}(0.5,1,1.5$ and $2 \mu \mathrm{M})$ for 12 , 24,48 and $72 \mathrm{~h}$ markedly increased NO production both in a dose- and time-dependent manner (Fig. 5C and D).

CuD triggers the activation of the mitochondrial pathway in gastric cancer cell lines. NO production is triggered by NO synthase (NOS). Apoptosis is triggered by intrinsic and extrinsic apoptotic pathways. Therefore, we evaluated the expression of iNOS and the mitochondrial pathway in AGS cells after treatment with $\operatorname{CuD}(0.5,1$ and $2 \mu \mathrm{M})$ for $24 \mathrm{~h}$. Our results revealed that $\mathrm{CuD}$ increased the expression level of iNOS, which triggered the generation of NO. CuD also increased Bax levels and reduced the expression of Bcl-2, triggering the activation of caspase-9, as well as the release of cytochrome $c$ in AGS cells (Fig. 6).

CuD inhibits Akt-mediated signalling pathway. The Akt pathway is involved in many processes associated with the poor prognosis of cancer, such as pro-survival, anti-apoptosis, metastases and chemotherapy resistance (11). CuD has been reported to inhibit Akt in schwannoma and meningioma cells (13). We found that incubation of AGS cells with $\mathrm{CuD}(0.5,1$ and $2 \mu \mathrm{M})$ for $24 \mathrm{~h}$ significantly reduced the levels of phosphorylated Akt, mTOR (a downstream protein of the Akt pathway) and ribosomal S6 protein (rS6) (Fig. 7). These data indicated that $\mathrm{CuD}$ targeted the Akt signalling pathway in gastric cancer cells.

$\mathrm{CuD}$ targets Akt and $\mathrm{iNOS}$. To confirm that the effects of $\mathrm{CuD}$ were dependent on Akt and iNOS, AGS cells were treated with SC79 $(4 \mu \mathrm{g} / \mathrm{ml})$ or L-canavanine $(1 \mathrm{mM})$ and $\mathrm{CuD}(2 \mu \mathrm{M})$ for $24 \mathrm{~h}$. We observed that both SC79 and L-canavanine reversed the antiproliferative and pro-apoptotic effects of $\mathrm{CuD}$ in AGS cells (Fig. 8). This indicated that $\mathrm{CuD}$ targeted Akt and iNOS.

Effects of $C u D$ on gastric cancer in a xenograft mouse model. The effects of $\mathrm{CuD}$ on gastric cancer were confirmed by an in vivo xenograft-mouse model. After tumours became palpable, the mice were treated with either $\mathrm{CuD}$ and/or SC79 for 20 days. We found that $\mathrm{CuD}$ induced tumour cell apoptosis and arrested tumour growth in vivo. In contrast, SC79 inhibited tumour cell apoptosis and augmented tumour growth. In addition, SC79 reversed the anti-growth effect of CuD (Fig. 9). 
A

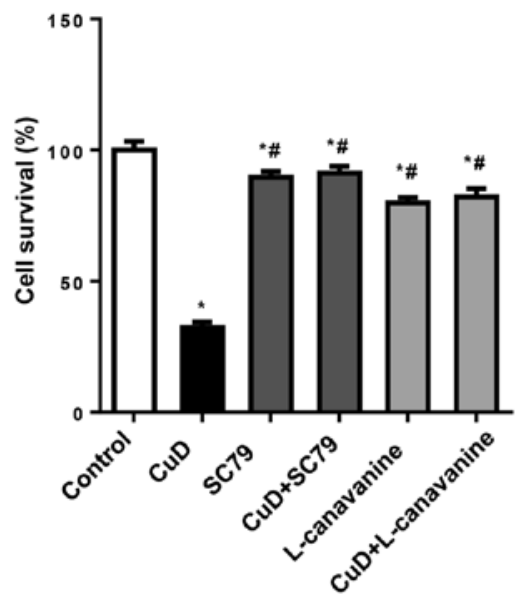

C

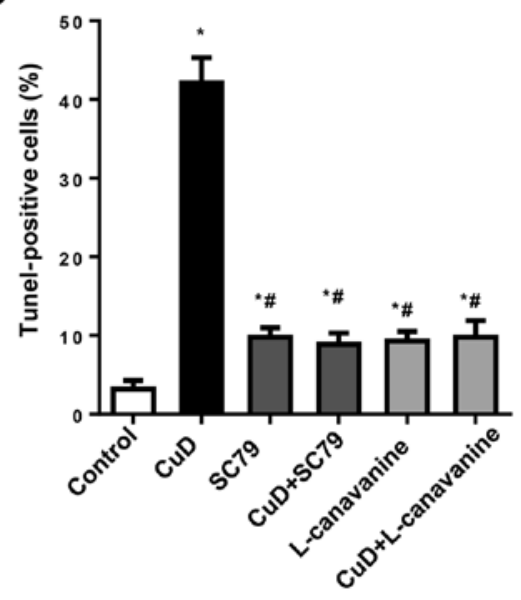

B
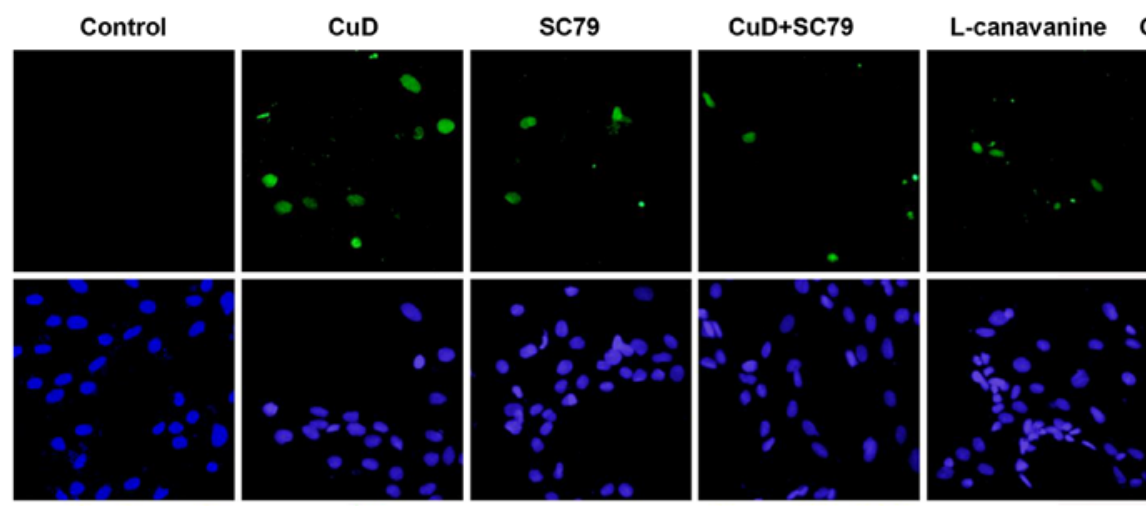

CuD+L-canavanine
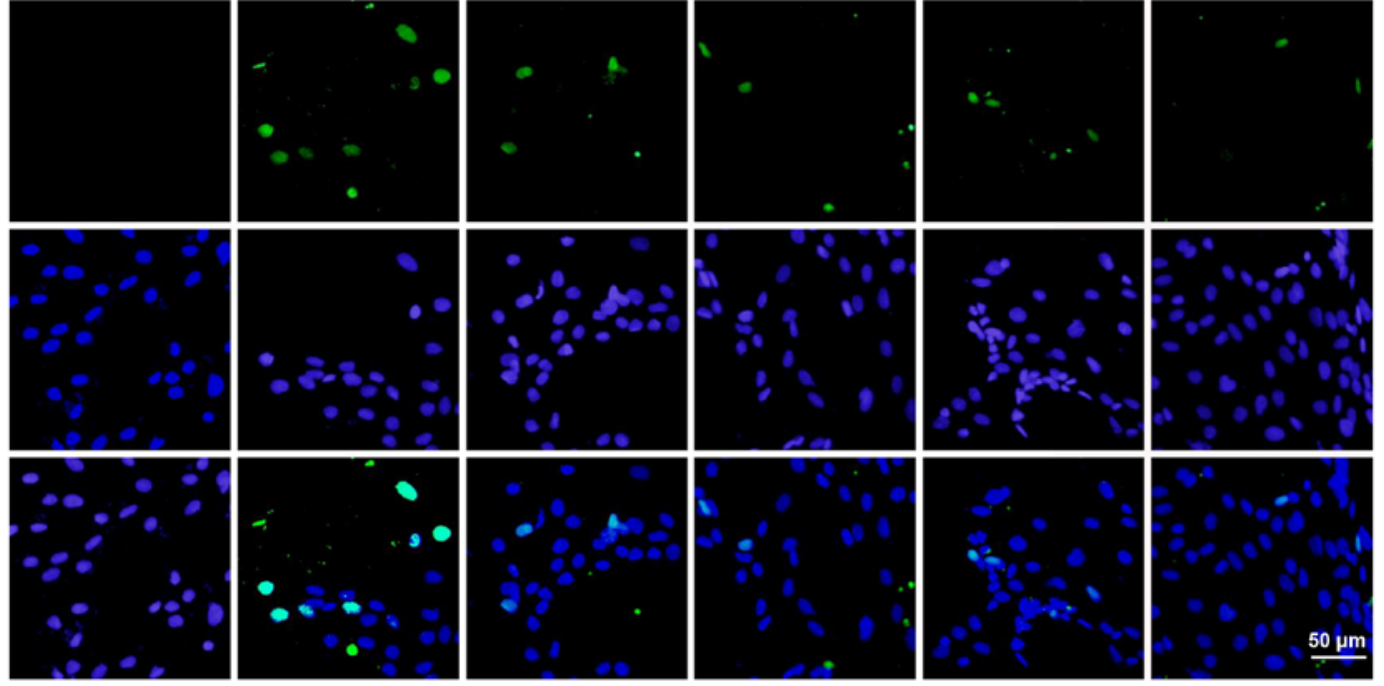

Figure 8. Akt and iNOS are the target of cucurbitacin D (CuD). (A) The effect of CuD $(2 \mu \mathrm{M}), \mathrm{SC} 79(4 \mu \mathrm{g} / \mathrm{ml})$, L-canavanine $(1 \mathrm{mM})$ on cell proliferation in the AGS cell line. (B and C) The effect of CuD $(2 \mu \mathrm{M})$, SC79 $(4 \mu \mathrm{g} / \mathrm{ml})$, and L-canavanine $(1 \mathrm{mM})$ on cell apoptosis in the AGS cell line. ${ }^{*} \mathrm{P}<0.05$ compared to control group; ${ }^{\text {}} \mathrm{P}<0.05$ compared to $\mathrm{CuD}$ group.

\section{Discussion}

Gastric cancer, one of most common malignant tumours and the second leading cause of cancer-related deaths worldwide, is the fourth most common cancer worldwide (14). Although the incidence of gastric cancer varies greatly among countries, in developing countries the incidence of gastric cancer is $>70 \%$ (3). The efficacy of chemotherapy in the treatment of local and metastatic gastric cancer is limited by chemoresistance, which results in treatment failure. Complementary and alternative medicine can be used to overcome these limitations. Plant derived natural products have attracted widespread attention because they are less toxic (15). In the present study, we found that cucurbitacin $\mathrm{D}(\mathrm{CuD})$ induced apoptosis and subsequently inhibited the growth of gastric cancer cells, making it a potential therapeutic agent for the treatment of gastric cancer.

Oxidative stress, characterized by the imbalance of ROS and anti-oxidants, has been implicated in the pathogenesis of several diseases (16). At lower concentrations, ROS are important signalling molecules involved in cellular proliferation, migration and apoptosis. Athigher concentrations, most ROS are harmful to cells due to the accumulation of irreversible damage to proteins, lipids and most importantly, to DNA, leading to mutations and cell death (17). Our functional experiment revealed that $\mathrm{CuD}$ significantly promoted ROS generation in gastric cancer cells.

Increased proliferation, diminished differentiation and reduced apoptosis are the features of tumour cells. Therefore, the most effective target in cancer therapy is promotion of apoptosis (18). The pro-apoptotic property of cucurbitacins makes them a potential antitumour agent $(8,15,19)$. In addition, $\mathrm{CuD}$ reportedly induced cell apoptosis in human $\mathrm{T}$ cell leukaemia (7), breast carcinoma (8), human endometrial and ovarian cancer cells (20) and cervical cancer (5). Our results indicated that $\mathrm{CuD}$ induced apoptosis in AGS, SNU1 and Hs746T gastric cancer cells. Mitochondrial membrane permeabilization (MMP) is closely associated with cancer cell death. Mitochondrial $\mathrm{Ca}^{2+}$ overload drives ROS generation, triggering MMP and release of pro-apoptotic factors, leading to cell apoptosis and death (21). Furthermore, high concentrations of NO derived from iNOS inhibit the expression of Bcl-2, 
A

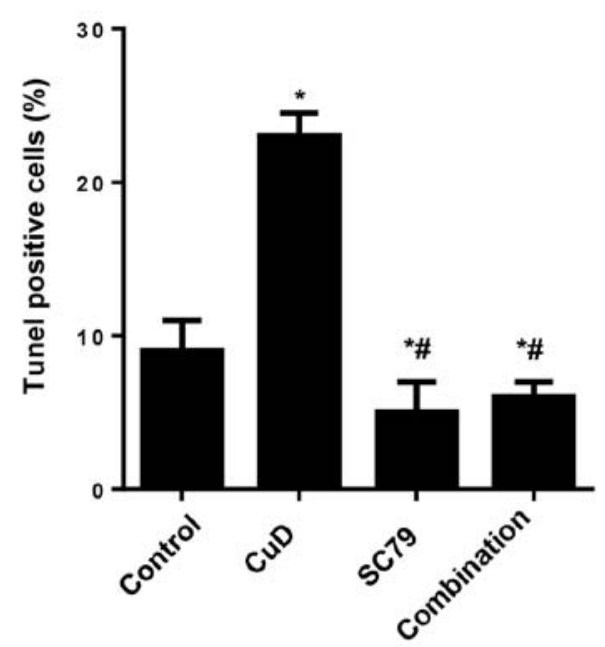

B

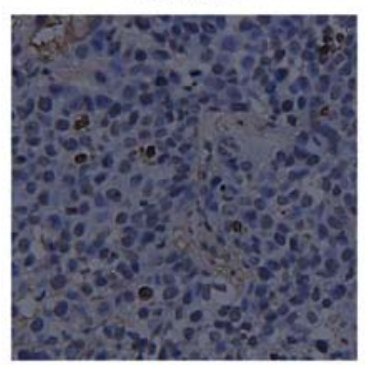

CuD

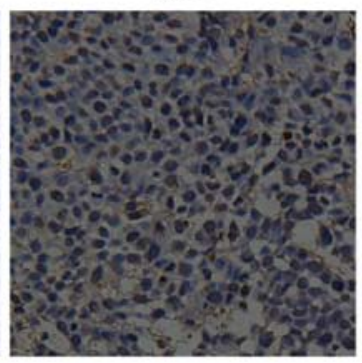

C

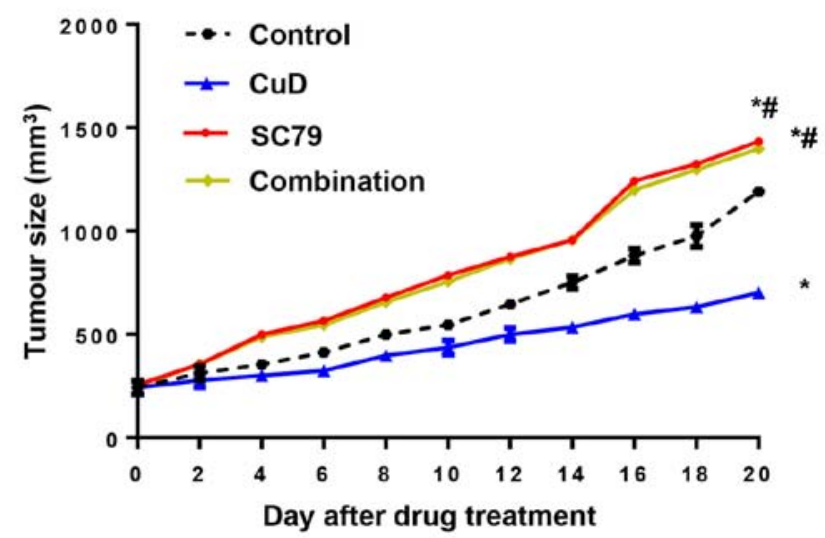

SC79
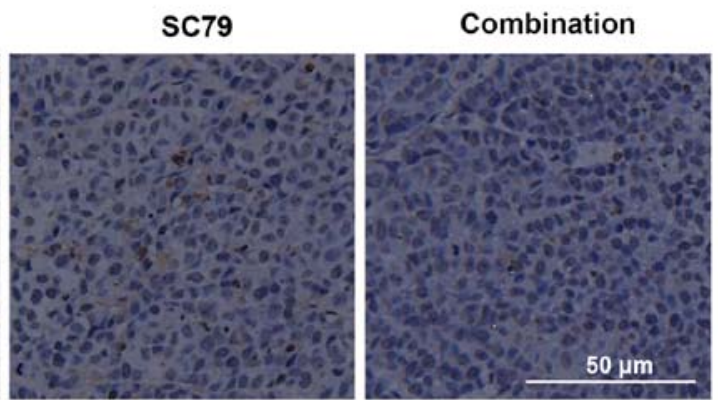

D

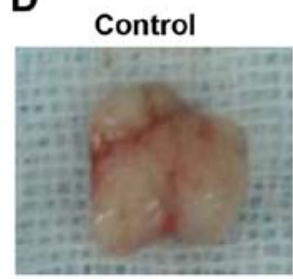

CuD

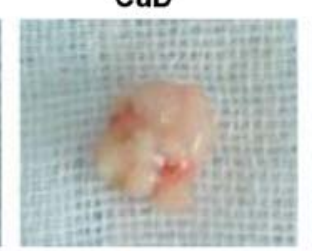

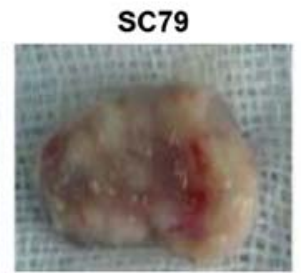

Combination

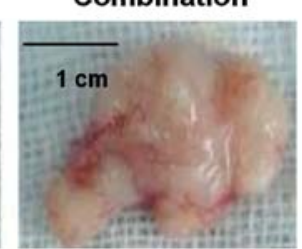

Figure 9. Cucurbitacin D (CuD) exerts anti-growth effect in an in vivo gastric cancer xenograft mouse model. (A and B) The effect of CuD (2 $\mu \mathrm{M})$ and/or SC79 $(4 \mu \mathrm{g} / \mathrm{ml})$ on cell apoptosis in gastric cancer xenografts (Tunel stain analysis). (C and D) The effect of CuD ( $2 \mu \mathrm{M})$ and/or SC79 (4 $\mu \mathrm{g} / \mathrm{ml})$ on tumour growth in gastric cancer xenografts. ${ }^{*} \mathrm{P}<0.05$ compared to the control group; ${ }^{\text {}} \mathrm{P}<0.05$ compared to the $\mathrm{CuD}$ group.

activate caspase and subsequently promote the release of cytochrome $c$, leading to cell death (22). In the present study, $\mathrm{CuD}$ raised MMP levels and increased iNOS expression and NO levels, which triggered modulation of $\mathrm{Bcl}-2$ proteins and cleavage of caspase- 9 and promoted cytochrome $c$ release from the mitochondria. Various oncogenic pathways participate in the progression of gastric cancers such as, nuclear factor- $\kappa \mathrm{B}, \mathrm{PI} 3 \mathrm{~K} / \mathrm{Akt}$ and Wnt/ $\beta$-catenin (18). Among these, Akt regulates cell survival, growth, metabolism and chemotherapy resistance (11). Furthermore, Akt regulates cell survival by targeting multiple downstream proteins. One downstream protein is mTOR, which can be activated through mTOR complex 1 (mTORC1). After activation, mTOR induces activity of ribosomal protein S6 kinase $\beta-1$ (S6K1), which promotes cell proliferation $(11,23)$. Alteration of the $\mathrm{Akt} / \mathrm{mTOR}$ pathway is the second most common cause of cancer in humans. Immunohistochemistry staining has shown that $74 \%$ of gastric cancers have high Akt expression, but genomic sequencing has demonstrated that $1-3 \%$ of gastric cancers have high Akt expression $(11,24)$. In the present study, $\mathrm{CuD}$ effectively inhibited Akt signalling in gastric cancer cells. Our in vivo study consistently demonstrated that $\mathrm{CuD}$ exerted anti-growth effects in the in vivo xenograft tumour model, however, those anti-growth effects could be reversed by an Akt activator.

In the present study, the caspase- 9 activity was assessed by the ratio of cleaved caspase- 9 to GAPDH, not the ratio of cleaved caspase-9 to total caspase-9. This may cause some inaccuracy. Secondly, whether $\mathrm{CuD}$ affected equally Akt and iNOS signalling was not clear. In addition, whether Akt and iNOS signalling are the only target of $\mathrm{CuD}$ anti-gastric cancer effects remain unknown. Future studies including a rescue assay to demonstrate that $\mathrm{CuD}$ induces apoptosis via inhibition of Akt and activation of the iNOS pathway are needed.

In conclusion, $\mathrm{CuD}$ inhibited gastric cancer in vitro and in vivo by inducing iNOS/NO signalling and suppressing the Akt pathway. 


\section{Acknowledgements}

Not applicable.

\section{Funding}

No funding was received.

\section{Availability of data and materials}

The datasets used during the present study are available from the corresponding author upon reasonable request.

\section{Authors' contributions}

ZYZ and ZLF conceived and designed the study. ZYZ, ZLF and WCF performed the experiments. ZLF and ZYZ wrote the manuscript. WCF reviewed and edited the manuscript. All authors read and approved the manuscript and agree to be accountable for all aspects of the research in ensuring that the accuracy or integrity of any part of the work are appropriately investigated and resolved.

\section{Ethics approval and consent to participate}

All animal experiments were performed according to the National Institutes of Health Guide for the Care and Use of Laboratory Animals (NIH Publications no. 8023, revised 1978). All animal experiments were approved by the Committee on the Use of Live Animals of The First Affiliated Hospital of Zhengzhou University.

\section{Consent for publication}

Not applicable.

\section{Competing interests}

The authors declare that they have no competing interests.

\section{References}

1. Takahashi T, Saikawa Y and Kitagawa Y: Gastric cancer: Current status of diagnosis and treatment. Cancers 5: 48-63, 2013.

2. Patel TN, Roy S and Ravi R: Gastric cancer and related epigenetic alterations. Ecancermedicalscience 11: 714, 2017.

3. Gastric Cancer Treatment $\left(\mathrm{PDQ}^{\circledR}\right)$ : Health Professional Version. In: PDQ Cancer Information Summaries, Bethesda, MD, 2002.

4. Hu B, El Hajj N, Sittler S, Lammert N, Barnes R and Meloni-Ehrig A: Gastric cancer: Classification, histology and application of molecular pathology. J Gastrointest Oncol 3: 251-261, 2012.

5. Sikander M, Hafeez BB, Malik S, Alsayari A, Halaweish FT, Yallapu MM, Chauhan SC and Jaggi M: Cucurbitacin D exhibits potent anti-cancer activity in cervical cancer. Sci Rep 6: 36594, 2016.

6. Wang Y, Sun Y, Wu Y and Zhang J: Cucurbitacin E inhibits osteosarcoma cells proliferation and invasion through attenuation of PI3K/AKT/mTOR signaling. Biosci Rep: Sep 21, 2016 (Epub ahead of print).
7. Nakanishi T, Song Y, He C, Wang D, Morita K, Tsukada J, Kanazawa T and Yoshida Y: Autophagy is associated with cucurbitacin D-induced apoptosis in human T cell leukemia cells. Med Oncol 33: 30, 2016.

8. Ku JM, Kim SR, Hong SH, Choi HS, Seo HS, Shin YC and Ko SG: Cucurbitacin D induces cell cycle arrest and apoptosis by inhibiting STAT3 and NF- $\kappa$ B signaling in doxorubicin-resistant human breast carcinoma (MCF7/ADR) cells. Mol Cell Biochem 409: 33-43, 2015.

9. Hall JA, Seedarala S, Rice N, Kopel L, Halaweish F and Blagg BS: Cucurbitacin D is a disruptor of the HSP90 chaperone machinery. J Nat Prod 78: 873-879, 2015.

10. Hudler P: Challenges of deciphering gastric cancer heterogeneity. World J Gastroenterol 21: 10510-10527, 2015

11. Tran P, Nguyen C and Klempner SJ: Targeting the Phosphatidylinositol-3-kinase pathway in gastric cancer: Can omics improve outcomes? Int Neurourol J 20 (Suppl 2): S131-S140, 2016

12. Fang WL, Huang KH, Lan YT, Lin CH, Chang SC, Chen MH, Chao Y, Lin WC, Lo SS, Li AF, et al: Mutations in PI3K/AKT pathway genes and amplifications of PIK3CA are associated with patterns of recurrence in gastric cancers. Oncotarget 7: 6201-6220, 2016

13. Spear SA, Burns SS, Oblinger JL, Ren Y, Pan L, Kinghorn AD, Welling DB and Chang LS: Natural compounds as potential treatments of NF2-deficient schwannoma and meningioma: Cucurbitacin D and goyazensolide. Otol Neurotol 34: 1519-1527, 2013.

14. Cheng XJ, Lin JC and Tu SP: Etiology and prevention of gastric cancer. Gastrointest Tumors 3: 25-36, 2016.

15. Lui VW, Yau DM, Wong EY, Ng YK, Lau CP, Ho Y, Chan JP, Hong B, Ho K, Cheung CS, et al: Cucurbitacin I elicits anoikis sensitization, inhibits cellular invasion and in vivo tumor formation ability of nasopharyngeal carcinoma cells. Carcinogenesis 30: 2085-2094, 2009.

16. Marengo B, Nitti M, Furfaro AL, Colla R, Ciucis CD, Marinari UM, Pronzato MA, Traverso N and Domenicotti C: Redox homeostasis and cellular antioxidant systems: Crucial players in cancer growth and therapy. Oxid Med Cell Longev 2016: 6235641, 2016.

17. Morry J, Ngamcherdtrakul W and Yantasee W: Oxidative stress in cancer and fibrosis: Opportunity for therapeutic intervention with antioxidant compounds, enzymes, and nanoparticles. Redox Biol 11: 240-253, 2017.

18. $\mathrm{Xu} \mathrm{W}$, Yang $\mathrm{Z}$ and $\mathrm{Lu} \mathrm{N}$ : Molecular targeted therapy for the treatment of gastric cancer. J Exp Clin Cancer Res 35: 1, 2016.

19. Ma G, Luo W, Lu J, Ma DL, Leung CH, Wang Y and Chen X: Cucurbitacin E induces caspase-dependent apoptosis and protective autophagy mediated by ROS in lung cancer cells. Chem Biol Interact 253: 1-9, 2016.

20. Ishii T, Kira N, Yoshida T and Narahara H: Cucurbitacin D induces growth inhibition, cell cycle arrest, and apoptosis in human endometrial and ovarian cancer cells. Tumour Biol 34: 285-291, 2013.

21. Chen LD, Liu ZH, Zhang LF, Yao JN and Wang CF: Sanggenon C induces apoptosis of colon cancer cells via inhibition of No production, iNOS expression and ROS activation of the mitochondrial pathway. Oncol Rep 38: 2123-2131, 2017.

22. Vannini F, Kashfi K and Nath N: The dual role of iNOS in cancer. Redox Biol 6: 334-343, 2015.

23. Sasaki T and Kuniyasu H: Significance of AKT in gastric cancer (Review). Int J Oncol 45: 2187-2192, 2014.

24. Almhanna K, Strosberg J and Malafa M: Targeting AKT protein kinase in gastric cancer. Anticancer Res 31: 4387-4392, 2011. International (CC BY-NC-ND 4.0) License. 